\title{
Molecular study of the dentin-pulp complex responses to caries progression
}

\author{
Yani Corvianindya Rahayu \\ Department of Oral Biology \\ University of Jember Faculty of Dentistry \\ Jember - Indonesia
}

\begin{abstract}
The dentin-pulp complex exhibits various responses to caries, including events of injury, defense, and repair. The overall responses dependent on pulp cell activity and the signaling processes, which regulate the behavior of these cells. The signals for tissue repair are thought to be mediated by dentin-bound growth factors released during caries progression. Growth factors are a key of molecules responsible for signaling a variety of cellular process following dental injury. The endogenous proteolytic enzymes (Matrix metalloproteinases, MMPs) present in dentin matrix might also participate in releasing bioactive molecule. Several members of the MMP family are found in the soft and hard tissue compartment of dentin-pulp complex. Their presumed role in many physiological process during the development and maintenance of the dentin-pulp complex, they may also contribute to the pathogenesis of dentin caries and the responses elicited by caries.
\end{abstract}

Key words: Dentin-pulp complex, Molecular events, Caries progression

Correspondence: Yani Corvianindya Rahayu, c/o: Bagian Biologi Oral, Fakultas Kedokteran Gigi Universitas Jember. Jln. Kalimantan 37 Jember 68121, Indonesia.

\section{INTRODUCTION}

The human dentition is indispensable for nutrition and physiology. The teeth have evolved for mastication of food. Caries is a common dental problem in which the dentin matrix is damaged. When the caries is deep and the dental pulp is exposed, the pulp has to be removed in many cases, resulting ultimately in loss of the tooth. ${ }^{1}$

The dentin-pulp complex comprises mineralized dentin and the vital soft tissues encased inside dentin, i.e. odontoblasts and pulp tissue. During caries progression, the dentinal minerals are dissolved and eventually the collagenous organic matrix is degraded. However, the exact mechanisms and enzymes responsible for the organic matrix breakdown remain unknown. ${ }^{2}$

The vitality of the dentin-pulp complex, both during tissue homeostasis and after injury, is dependent on pulp cell activity and the signaling processes, which regulate the behavior of these cells. Research, particularly over the last ten to fifteen years, has led to a better understanding of the molecular control of cellular behavior. Growth factors play a pivotal role in signaling the events of tissue formation and repair in the dentin-pulp complex. Sequestration of growth factors in the dentin matrix during tissue formation provides a pool of these molecules, which may be released during injury and contribute to signaling of reparative events. ${ }^{3}$

It is obvious that much information is still lacking in our understanding of how the immune system and other components of the dentin/pulp complex communicate to mount defense, damage, and repair of the dentin/pulp complex. Most reports in this area are descriptive, and information on molecular mechanisms regulating cellular responses is limited. Future research should be extended to delineate the profile of bioactive molecules such as cytokines that sequentially regulate the immunological reactions of the dental pulp. ${ }^{2,4}$

This article will review current knowledge of the understanding the molecular events underlying the dentinpulp complex responses to carious progression. It will also describe the roles of some enzymes and bioactive molecules in dentin matrix might participate in degradative events of caries processes.

\section{Biology of dentin-pulp complex}

Teeth are composed principally of dentin, and the pulp is its formative tissue. Biologically the two are inseparable, although mature teeth can functions adequately in the absence of the pulp, such as when the dental endodontic and restorations procedures and formed and successfully concluded. Normally, however the pulp of the tooth is vital, active, and responsive to its environment, a responsiveness that is reflected not only as an inherent change in its structure, but also as an alterations in the form and structure of the dentin it produces. The presence of the pulp also imparts a sensitively to the dentin, a sensitivity that in physiologic terms may be distinctive to the body. Because of this intimate relationship the dentin and the pulp are generally considered as a complex, the dentinpulp complex. ${ }^{5}$

Biologically and developmentally, pulp and dentin function as a complex and may be regarded as one tissue. 
Dentinal fluid movement, resulting in hydrodynamic activation of pulpal A-delta nerve fibers and causing dentin sensitivity, is a common example of functional coupling of the tissues. Both tissue are derived from the dental papilla, and development of two tissues is closely related. The structure and composition of dentin matrix, and of the dentinal tubules, are key influences in the process of bacterial invasion of dentinal tubules. ${ }^{5,6}$

The dentin extra cellular framework, into and on which the minerals are embedded, is also called organic matrix, with reference to its role as a template for mineralization and its presumable regulative activity on mineralization. Collagen comprise over $90 \%$ of the dentin organic matrix, type I collagen being the most abundant collagen. Type III collagen expressed and synthesized by human odontoblast, it has been observed in intratubular dentin and predentin as well as inside the dentinal tubules. ${ }^{7}$

Many non-collagenous ECM protein are considered important in actively promoting and controlling yhe mineralization of collagen fibrils and crystal growth during dentin formation. Dentin contains large amounts of dentin sialoprotein and dentin phosphoproteins compared to bone. Dentin non-collagenous ECM also contains plasma protein and several growth factors. ${ }^{2,8}$

\section{Responses of dentin-pulp complex to caries}

The dentin-pulp complex reacts to a variety of bacterial and nonbacterial stimuli. Most common and perhaps most typical of the bacterial-related reactions is the manner in which the pulp responds to dental caries. Microscopically the reaction is identified in the pulp by a disruption of the odontoblastic layer and an edema and inflammatory round cell infiltration of the pulp core. Since the bacteria have not as yet entered the pulp, it thought that these changes represent an immunologic response to bacterial by products or components that diffuse into the pulp from carious lesion via dentinal tubules. ${ }^{4,7}$

The overall response of the tooth to injury, such as dental caries, represents the complex interplay between injury, defense, and regenerative process (Figure 1). It is important to recognize that the interplay and relative balance among these processes will be primary determinant of tissue vitality and tooth survival. ${ }^{3}$

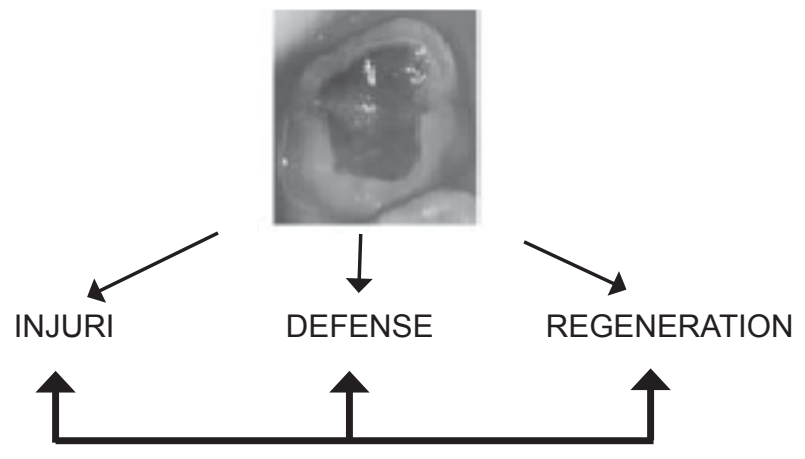

Figure 1. The overall response of the tooth to injury, such dental caries, represents the complex interplay among injury, defense and regeneration. ${ }^{3}$
Bacterial invasion of dentinal tubules commonly occurs when dentin is exposed following a breach in the integrity of the overlying enamel or cementum. Bacterial products diffuse through the dentinal tubule toward the pulp and evoke inflammatory changes in the dentin-pulp complex. ${ }^{6}$

Defense encompassed a variety of local tissue and systemic responses to counteract the injury. These will include local inflammation within the tissues as well as local activation of immune defense reactions, which may trigger broader systemic immune responses. T lymphocytes and macrophages have been proposed to be important in the immunosurveillance of the pulp, and may be important in the initiation of pulpal specific immunity following exposure to protein antigens. $\mathrm{B}$ and $\mathrm{T}$ lymphocytes are increases in numbers of these cells have been correlated with increasing lesion depth in caries. ${ }^{4,6}$

Macrophages, lymphocytes and antibody-producing plasma cells (immunocompetent cells) are predominant cell types notes in the pulp prior to its actual exposure (direct invasion by microorganism), polymorphouclear cells are also present. The immunocompetent cells within the pulp appear tobe enganged in antibody production. Various antibodies (IgG, IgE, IgA) have been identified in pulps affected by caries. $4,6,7$

Dental injury perhaps represents a special situation in that the cellular pulp is enclosed by a rigid, mineralized, tissue shell and thus significant enamel and dentin matrix degradation can ensue before the disease process reaches the pulp. The tubular structure of dentin confers permeability properties on the tissue, and bacterial metabolic products from the carious process and dental tissue matrix degradation products may diffuse down the dentinal tubules and invoke cellular responses. ${ }^{3}$

Dentin permeability, influenced by both the density and the diameter of the dentinal tubules, has a major effect on the extent of injury to the dentin-pulp complex cells by the noxious component. Dentin permeability may be decrease by tertiary dentin formation and by obliteration of the tubules, through either physiological dentin sclerosis that is evenly distributed in dentin or reactive dentin sclerosis observed in carious teeth beneath and next to the caries lesion. Reactive dentin sclerosis may result from the acceleration of intratubular dentinogenesis, but also by precipitation of dissolved minerals. ${ }^{9,10}$

Deposition of tertiary dentin at the site of injury is generally intermittent, reflecting the intermittent nature of the caries attact. The processes of reactionary and reparative dentinogenesis at dental caries for secretion of tertiary dentin matrices, which either increase the dentin barrier between the site of injury and the underlying cells in the unexposed pulp., 3

\section{Growth factors as key mediators}

Growth factors are peptide molecules that transmit signals between cell functioning as stimulators and/or inhibitors of growth as well as modulators of differentiation state amongst other roles. As such, they play a central role 
in controlling cell behavior and activity. Growth factors are a key of molecules responsible for signaling a variety of cellular process following dental injury. They play a central role in signaling various aspects of tooth morphogenesis, differentiation, tissue regeneration, and events associated with the initial injury to the tissue and subsequent defense reactions also impact on their activities. Importantly, they provide the basis to understand the biological mechanisms of tissue regeneration in dentin-pulp complex. ${ }^{3}$

Growth factors may be released from the dentin matrix as a result of injury events. During caries, diffusion of acidic plaque bacterial metabolites into the tissue will lead to demineralization and release of soluble extracelluler matrix component, including growth factors (Figure 2).

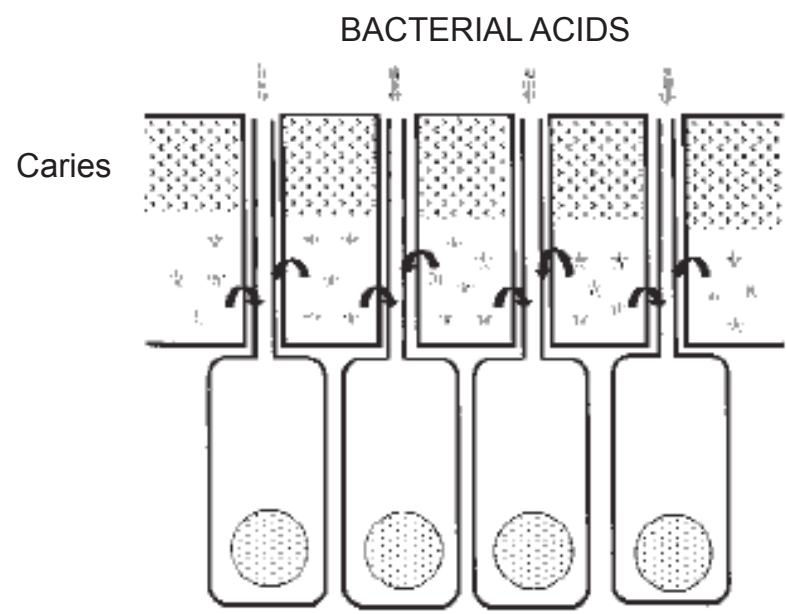

Figure 2. Mehanisms of release of growth factors (grey stars) from dentin matrix and their subsequent diffusion along the dentinal tubules to interact with the odontoblast and pulp cells. ${ }^{3}$

It has now been possible to identify a number of growth factors in dentin matrix, which while quantitatively minor component, may nave potent biological effects. A key family of growth factors, which have been identified in dentin, are members of the TGF- $\beta$ (transforming growth factor- $\beta$ ) family. It have been implicated in signaling of odontoblast differentiation. Odontoblast express all three isoforms of TGF (TGF-1, -2 , and -3), but only TGF- $\beta$ becomes sequestrated within the matrix and may help to dampen the inflammatory response in the pulp. ${ }^{11}$

A variety of cytokines (cell-signaling molecules associated with inflammatory and immune reactions) recognized to be associated with pulp responses to dental caries. In caries lesion progression, the initial diffusion of acid into the tissue will be followed by diffusion of plaque bacteria-derived proteolytic enzyme, which will degrade the insoluble dentin matrix. Release of the associated growth factors will further supplement the soluble pools of growth factors from the tissue. Thus, the degradative events of caries may be important in releasing and unmasking bioactive molecules, which have potential to signal many of defense and repair events in the tooth. The endogenous proteolytic enzymes (Matrix metalloproteinases, MMPs) present in dentin matrix might also participate in releasing bioactive molecule, even in the absence of bacteria, if their normal regulatory control becomes compromised in pathologic conditions. ${ }^{3}$

\section{The roles of MMPs in carious progressions}

Matrix metalloproteinases (MMPs) have been detected in both soft and hard tissue compartment of the dentinpulp complex, and their activity has been suggested to be involved in various physiological processes of the formation and maintenance of the dentin-pulp complex.

MMPs, a family of endopeptidases capable of degrading in concert virtually all extra cellular matrix components, are expressed during normal dentin-pulp complex formation and maintenance. MMP activity has also been suggested to contribute to the organic matrix degradation during dentin caries progression and to the repair and defense reactions elicited by caries in the dentin-pulp complex cells. ${ }^{2}$

During early tooth development, dental mesenchymal cells are observed to express at least MMP-1, -2, -3 and -9 . MMP-3 suggested to be involved in the organization of the dentin matrix constituents before mineralization inside the dentinal tubules. After the completion of tooth development, odontoblast synthesize MMP-2, -8, -9 and20. These MMPs are also synthesized in human pulp tissue. Some of the MMPs involved in dentin formation become embedded into mineralized dentin, since a latent enzyme with collagenolytic activity as well as gelatinolytic metalloproteinases, specificallyMMP-2. ${ }^{12}$

The MMPs of the dentin-pulp complex have been suggested to be involved in pathological processes, including dentin ECM destruction during caries progression and activation of dentinal growth factors due to external non-physiological stimuli, the formation of tertiary dentin, tissue destruction in pulpal inflammation, and breakdown of demineralized collagen in the adhesive layer of dental composite restorations. ${ }^{2,9}$

\section{DISCUSSION}

The dentin-pulp complex exhibits various responses to caries, including events of injury, defense and repair. These responses are reactions in the vital tissues of the tooth in combination with physico-chemical processes involving the mineral phase of the tooth. ${ }^{2,9}$

Bacterial acids and other metabolites, even though diluted and mixed with dentinal constituents, are noxious to the cells of the dentin-pulp complex, and due to the tubular structure of dentin. Caries induce changes in the morphology and enzymatic activity of odontoblast cells as well as a decrease in predentin width and accumulation of cells into the cell-free zone. The first sign of pulpal defense is a local inflammation under the site of irritation. 
The inflammatory cells infiltrate consist mainly of T-lymphocytes, macrophage, neutrophils, B-lymphocytes and plasma cells. ${ }^{13,14}$

Proteolytic enzyme of host and/or microbial origin have been detected in carious dentin, especially in the dentinal tubules. Cariogenic microbes are required for caries development, but evidence for their collagenolytic or even proteolytic activity is inconclusive. ${ }^{2}$

The matrix metalloproteinase family is mainly responsible for the degradation of the structural macromolecules of the interstitial connective tissue in the extracelluler milieu during physiological ECM turnover and reorganization in human body, presumably including the dentin-pulp complex. As in other tissues, MMP activity may also be altered in pathological conditions of the dentinpulp complex. MMPs may contribute to the degradation of the dentin organic matrix during caries progression, but also to the response reactions to caries in the dentin-pulp complex. ${ }^{2,9}$

Kamal, 1997 studied that the response of Ia antigenexpressing cells to carious irritants triggers the defense reactions of the pulp. The intensity of the defense reaction may be correlated with the permeability of carious dentin. ${ }^{15}$

The signals for tissue repair are thought to be mediated by dentin-bound growth factors released during caries progression. The processes of reactionary and reparative dentinogenesis at sites of dental injury are responsible for secretion of tertiary dentin matrices, which either increase the dentin barrier between the site of injury and the underlying cells in the unexposed pulp or provide a dentin bridge across the exposed pulp. Growth factors may be a key molecules in the signaling of the biological even responsible for these processes. ${ }^{13}$

A number of reports of the transdentinal or direct application of TGF- $\beta-1$ and BMP-7 to the odontoblast of unexposed pulp in cultured tooth shown ability of these GF to signal reactionary dentinogenesis. These findings suggest exciting opportunities for biologically based therapeutic approaches to dental tissue repair as well as providing valuable insights into how natural regenerative processes may be operating in the tooth. ${ }^{3,16}$

Experimental investigations in mature teeth have shown that a network of extra cellular matrix molecules and growth factors signal tertiary dentinogenesis. Application of dentin matrix components or growth factors in deep dentinal cavities stimulated up-regulation of biosynthetic activity of primary odontoblasts (reactionary dentin formation). ${ }^{16}$

Lee et al., ${ }^{17}$ analyzed tissue morphology and dentin matrix protein distribution in non-carious teeth and in teeth with enamel and dentin caries to understand the molecular events underlying the dentin-pulp complex responses to carious progression. Dentin matrix proteins analyzed included collagen type I, phosphophoryn (PP) and dentin sialoprotein (DSP), all of which play decisive roles in the dentin mineralization process. The carious lesions stimulate the dentin-pulp complex to actively synthesize collagen type I, PP and DSP proteins. This response to carious lesions is likely to provide a basis for reparative and/or reactionary dentin formation.

Recent advances in stem cell biology and gene therapy technology have provided the great potential of adult stem cells for therapeutic use in regeneration of lost tissue due to diseases including cancer, trauma, and even caries. Dental pulp tissues harbor mesenchymal stem/progenitor cells and have potential to regenerate and/or repair dentin-pulp complex after injury such as caries. ${ }^{18}$

The vitality of the dentin-pulp complex, both during tissue homeostasis and after injury, is dependent on pulp cell activity and the signaling processes, which regulate the behavior of these cells. The processes of reactionary and reparative dentinogenesis at sites of dental injury are responsible for secretion of tertiary dentin matrices, which either increase the dentin barrier between the site of injury and the underlying cells in the unexposed pulp or provide a dentin bridge across the exposed pulp. Growth factors may be a key molecules in the signaling of the biological even responsible for these processes.

The degradation of the collagenous organic matrix of dentin during caries progression is an enzymatic process. Several members of the MMP family are found in the soft and hard tissue compartment of dentin-pulp complex. Their presumed role in many physiological process during the development and maintenance of the dentin-pulp complex, they may also contribute to the pathogenesis of dentin caries and the responses elicited by caries.

\section{REFERENCES}

1. Nakhasima M. Bone morphogenetic proteins in dentin regeneration for potential use in endodontic therapy. Cytokine Growth Factor Rev 2005 Jun;16:369-76.

2. Sulkala, M. Matrix metalloproteinase (MMPs) in dentin-pulp complex of healthy and carious teeth. 2004. Available from http://hercules. oulu.fi/isbn9514274598.htm. Accessed November 28, 2006.

3. Smith, AJ. Vitality of the dentin-pulp complex in health and disease: growth factors as key mediators. J Dent Educ 2003 Jun; 67:678-89

4. Jontell M, Okiji T. Immune defense mechanisms of the dental pulp. Crit Rev Oral Biol Med. 1998; 9(2):179-200.

5. Roth GI, Calmes R. Oral biology. $1^{\text {st }}$ ed. London: The CV Mosby Company; 1981. p. 119-37.

6. Love RM, Jenkinson HF. Invasion of dentinal tubules by oral bacteria. Crit Rev Oral Biol Med 2002; 13(2):171-83.

7. Waltimo J, Risteli L, Risteli J. Altered collagen expression in human dentin : increased reactivity of type III and presence of type VI in dentinogenesis imperfecta as revealed by immunoelectron microscopy. J Histochem Cytochem 1994; 42:1553-63.

8. Linde A, Goldberg M. Dentinogenesis. Crit Rev Oral Biol Med 1993; 4:679-708.

9. Pashley DH. Dynamic of the pulpo-dentin complex. Crit Rev Oral Biol Med 1996; 7:104-33.

10. Arnold WH, Konopka S, Gaengler P. Qualitative and quantitative assessment of intratubular dentin formation in human carious lesion. Calsif Tissue Int 2001; 69:268-73.

11. Cassidy N. Comparative analysis of TGF-B1-3 in human and rabbit dentine matrices. Arch Oral Biol 1997; 4(2):19-23. 
12. Palosaari H, Permington CJ, Larmas M, Edwards DR. Expression profile of MMPs and tissue inhibitors of MMPs in mature human odontoblast and pulp tissue. Eur J Oral Sci. 2003; 111:117-27.

13. Magloire H, Bouvier M, Joffre A. Odontoblast response under carious lesions. Proc Finn Dent Soc 1992; 88(suppl I):257-74.

14. Izumi T, Kobayashi I, Okamura K, Sakai H. Immunohistochemical study on the immunocompetent cells on the pulp in human non-carious \& carious teeth. Arch Oral Biol 1995; 40:609-14.

15. Kamal AM, Okiji T, Kawashima N, Suda H. Defense responses of dentin/pulp complex to experimentally induced caries in rat molars: an immunohistochemical study on kinetics of pulpal Ia
Antigen-expressing cells and macrophages. J Endod 1997 Feb; 23(2):115-20.

16. Tziafas D. The future role of molecular approach to pulp-dentinal regeneration. Caries Research 2004; 38:314-20.

17. Lee YL, Liu J, Clarkson BH, Lin CP, Godovikova V, Ritchie HH. Dentin-pulp complex responses to carious lesions. Caries Research 2006; 40:256-64.

18. Nakhasima M, Iohara K, Zheng L. Gene therapy for dentin regeneration with bone morphogenetic proteins. Curr Gene Ther 2006 Oct; 6(5):551-60. 